Research Article

\title{
Tripolarizated MIMO: Experimental Analysis and Modeling of Channel Properties in Both Indoor and Outdoor Scenarios
}

\author{
Pan Tang $\mathbb{D}^{1}{ }^{1}$ Jianhua Zhang $\left(\mathbb{D},{ }^{1}\right.$ Zuolong Ying, ${ }^{1}$ Yuxiang Zhang, ${ }^{1}$ Lei Tian, ${ }^{1}$ Zihang Qi, ${ }^{2}$ \\ and Xiuping $\mathrm{Li}^{2}$ \\ ${ }^{1}$ State Key Lab of Networking and Switching Technology, Beijing University of Posts and Telecommunications, \\ Beijing 100876, China \\ ${ }^{2}$ School of Electronic Engineering, Beijing University of Posts and Telecommunications, Beijing 100876, China
}

Correspondence should be addressed to Jianhua Zhang; jhzhang@bupt.edu.cn

Received 4 June 2021; Revised 13 August 2021; Accepted 11 September 2021; Published 28 September 2021

Academic Editor: Mourad Nedil

Copyright (c) 2021 Pan Tang et al. This is an open access article distributed under the Creative Commons Attribution License, which permits unrestricted use, distribution, and reproduction in any medium, provided the original work is properly cited.

The tripolarized MIMO system can provide one more degree of freedom and have a more compacted size over a dual-polarized MIMO system, which is attractive for high-capacity wireless communication systems. In this paper, we analyze and model channel properties for tripolarized MIMO systems based on experimental channel measurements in typical indoor and outdoor scenarios. Firstly, channel measurement campaigns in the laboratory and the Urban Micro (UMi) scenarios on sub-6 GHz bands are presented. Then, based on measured data, path loss, delay spread (DS), and cross-polarization discrimination (XPD) for 9 polarization combinations are analyzed and modeled in a statistical way. Statistical results of these channel properties are also given. It is observed that channel properties of both large-scale fading and small-scale fading depend strongly on the polarization direction. Furthermore, we evaluate the performance of tripolarized MIMO systems by analyzing the Demmel condition number and channel capacity gain (CG). For both the indoor and the outdoor scenarios, it is found that colocated tripolarized antenna can bring a nearly threefold CG with respect to the unipolarized one. These results can give good insights into the design and evaluation of tripolarized MIMO systems.

\section{Introduction}

In February 2020, the international telecommunication union (ITU) started the research towards 2030 and future sixth generation $(6 \mathrm{G})$. This indicates that $6 \mathrm{G}$ research has been evolved in the standardization plan of ITU. Until now, people have proposed a lot of opinions about $6 \mathrm{G}$ visions and enabling technologies [1-3]. For example, there are emerging application and new business, such as holographic type communications, multisense networks, time engineered applications, and critical infrastructure [4]. Also, 6G would expand in all aspects, e.g., the data rate, latency, spectral efficiency, and coverage, on the basis of fifth generation $(5 G)[5,6]$. To make $6 \mathrm{G}$ blueprint into reality, more communication resources (such as that in space, frequency, and time domains), new transmission technologies, and new network structure may be utilized [7].
Multiple-input-multiple-output (MIMO) technology has been widely used in mobile communications, which can increase data rates and improve the quality of wireless transmission by exploiting spatial diversity $[8,9]$. Without polarization, interantenna spacing has to be large enough to decrease interantenna correlation in MIMO systems. However, environmental considerations and tower loads place a limit on the size of the antenna array and the number of their antenna elements [10]. Thus, dual-polarized antennas have been widely deployed as they can decrease spacing needs and tower loads $[11,12]$. Furthermore, it was shown that using a tripolarized antenna can get an extra factor of three in channel capacity, relative to the conventional limit using dual-polarized radio signals [13]. Since tripolarized antennas can provide one more degree of freedom and save space, tripolarized MIMO can be an efficient technology to improve the data rate and spectral 
efficiency $[14,15]$. Especially, the tripolarized antenna is potential to be used in massive MIMO systems, which would deploy a large number of antenna elements and take up a large space.

Channel is the medium, in which the radio wave propagates, between the transmitter (TX) and the receiver (RX). And, channel properties determine the performance limit of wireless communication systems [16]. In a tripolarized MIMO channel, a polarized wave may experience polarization rotation due to scattering [17]. Correspondingly, channel correlation is decreased and channel capacity gain is also reduced. Furthermore, channel properties, e.g., large-scale fading properties and small-scale fading properties, in different polarization directions, may be different. Thus, having a deep understanding of tripolarized MIMO channel properties in the real world is one of the preconditions for the design and evaluation of tripolarized MIMO systems. In the following, we would give a review of research on channel properties for tripolarized MIMO systems.

1.1. Literature Review. In 2001, the idea of using three components of the electrical field was first proposed in [13]. Then, both theoretical and experimental research on channel properties for tripolarized MIMO systems was given. In [18], eigenvalue analysis of the channel matrix was given based on tripolarized MIMO channel measurements in the frequency range of 2.17 to $2.20 \mathrm{GHz}$ in an anechoic chamber. In $[19,20]$, theoretical channel models and simulations were used to investigate channel capacity of three dipole antennas with tripolarization. Furthermore, tripolarized MIMO channel measurements were performed in a controlled richscattering environment [14]. It was obtained that there is a threefold increase in channel capacity in simulation and experiment employing arrays of tripolarized dipole antennas. In [21], based on trip-polarized MIMO channel measurements in a corridor-to-office (C2O) scenario at $3.6 \mathrm{GHz}$, Ricean K-factor, cross-polarization discrimination (XPD), and correlation coefficients were analyzed. In [22], tripolarized MIMO channel measurements at $3.6 \mathrm{GHz}$ were presented. The authors analyzed the XPD and gave its statistical results. Also, correlation coefficients between all subchannels were measured and observed to be low. In $[15,23]$, authors compared channel properties, including XPD, Ricean K-factor, correlation coefficient, and channel capacity gain (CG), in a nonlossy reverberation chamber, a lossy chamber, and a corridor, and an office room. It was found that the effects of polarization coupling and transition depend strongly on the scattering characteristics of the environments.

In addition to the indoor scenario, an outdoor-to-indoor scenario was also measured at $3.5 \mathrm{GHz}$ by using a dualpolarized antenna at TX and a tripolarized antenna at RX [24]. Path loss for each of subchannels was analyzed and modeled. Furthermore, a measurement campaign in an urban microcell (UMi) scenario was presented in [25]. Besides, XPD, correlation coefficient, and CG were analyzed. Generally, we can see that the current research on channel properties for tripolarized MIMO systems mainly focuses on the analysis of XPD, correlation coefficient, and CG. The investigation on channel properties in different polarization directions is few. Also, most tripolarized MIMO channel measurements were conducted in indoor scenarios, while measurements in outdoor scenarios are scarce.

1.2. Contributions of This Paper. In this paper, we would give a relatively comprehensive investigation of channel properties for tripolarized MIMO systems. Channel measurements in both indoor and outdoor scenarios are conducted in the real world by using a virtual trip-polarized MIMO channel sounder to provide channel raw data. Based on measured data, channel properties are analyzed and modeled. Also, the system performance for tripolarized MIMO communications is evaluated. The main contributions of this paper are as follows:

(i) Based on a virtual tripolarized MIMO channel sounder, we conduct tripolarized MIMO channel measurements in the laboratory and the UMi scenarios. Also, both line-of-sight (LOS) and non-lineof-sight (NLOS) conditions are measured. This is a supplement to tripolarized MIMO channel measurements.

(ii) Based on measured channel raw data in the laboratory and the UMi scenarios, we analyze channel properties of large-scale fading and small-scale fading for tripolarized MIMO systems, including path loss, DS, and XPD. Furthermore, channel properties for different polarization combinations are compared and modeled in a statistical method. The fitting parameters of channel models are also given. This can provide proof of modeling the polarization dependency of channel properties in channel modeling for tripolarized MIMO systems.

(iii) $\mathrm{We}$ investigate the performance of tripolarized MIMO systems based on experimental measurements in the real world. Furthermore, the discrepancy of the system performance in different environments is analyzed and explored from the perspective of radio wave propagation. Especially, it is demonstrated that 3 independent subchannels can be supported by tripolarized MIMO systems in both indoor and outdoor scenarios.

1.3. Paper Organization. The rest of this paper is organized as follows. Section 2 introduces measurement setting and measurement procedures. Section 3 shows the analysis and modeling of channel properties for tripolarized MIMO systems. The system performance is evaluated in Section 4 by analyzing the Demmel condition number and channel capacity gain. Finally, Section 5 concludes this paper.

\section{Tripolarized MIMO Channel Measurements}

Channel measurement is a popular method of characterizing channels. It is also the prerequisite for building statistical channel models [26]. In this paper, we would present 
tripolarized MIMO channel measurements in the laboratory and the UMi scenarios. In the following, details about the system setting and measurement procedures would be given.

2.1. System Setting. In measurements, a correlation-based wideband channel sounder is used. Its structure is shown in Figure 1. At the TX side, a pseudonoise (PN) sequence with a length of 127 is first generated in the baseband. Then, the PN sequence is modulated using binary phase shift keying (BPSK). The center frequency is set to $3.5 \mathrm{GHz}$ and $4.9 \mathrm{GHz}$ in the laboratory and the UMi scenarios (since 5G network was built in our campus, the signal interference at $3.5 \mathrm{GHz}$ was strong in the UMi scenario; thus, we set the center frequency to $4.9 \mathrm{GHz}$ during measuring in the UMi scenario, which is also an important $5 \mathrm{G}$ band), respectively. And, the measured bandwidth is $100 \mathrm{MHz}$. To make the received signal has a large signal to noise (SNR), the PN signal is amplified through a power amplifier with a gain of $43 \mathrm{~dB}$. A dipole antenna is used to transmit the PN signal into space. At the RX side, a dipole antenna is used to receive the signal. To characterize the tripolarized MIMO channel, we rotate the dipole antenna in three orthogonal polarization directions to create virtual $3 \times 3$ MIMO channels [22]. Figure 2 shows the rotating positions of colocated antennas. Position 1 and position 2 are two orthogonal horizontal polarization, while position 3 is the vertical polarization. Furthermore, the dipole antenna in the position 1 points to the propagation direction between the TX and the RX, see Figure 3. In each measurement, TX and RX antennas are fixed in one of these three positions. Thus, there are totally 9 combinations. In the end, a spectrum analyzer (Keysight N9030A) is used to collect channel raw data. The detailed system settings are shown in Table 1.

2.2. Measurement Procedures. The indoor measurement was conducted in a laboratory. As shown in Figure 3, the laboratory is a closed room where there are some desks and metal boxes. Two dipoles are separately fixed in the tripods. Furthermore, the tripod at the TX side is put on the metal box to create a height difference between the TX and the RX, which makes the measured scenario be more realistic. The heights of the TX antenna and the RX antenna are $2.28 \mathrm{~m}$ and $1.28 \mathrm{~m}$, respectively. The measurement positions are plotted in Figure 4. There are 8 LOS positions and 8 NLOS positions. The distance between adjacent measurement positions is $0.5 \mathrm{~m}$. In measurements, the position of the TX is fixed while the RX moves to the next measurement position after measuring a $3 \times 3$ MIMO channel data. Also, people in the laboratory kept still to make sure the channel is stationary during measurements.

The outdoor measurement was conducted in an UMi scenario on the campus of the Beijing University of Posts and Telecommunications. Figure 5 shows the views of the TX antenna and the RX antenna. We can see that the TX tripod is fixed on the roof of a science hall, which is surrounded by higher buildings. And, the RX tripod is put on the ground. Buildings beside on both sides of the road make the scenario be like a canyon, which results in a rich- scattering scenario. The heights of the TX and the RX are $13.4 \mathrm{~m}$ and $1.7 \mathrm{~m}$, respectively. During measuring, the moving direction of the RX is parallel to the road. In each round of measurement, there is no pedestrian so that we assume the channel is also stationary. The placement method of dipole antennas is the same as that in the indoor measurements, as shown in Figure 3. Figure 6 shows the distribution of measurement positions in the UMi scenario. There are 20 LOS measurement positions and 20 NLOS measurement positions. The distance between the adjacent positions is $1 \mathrm{~m}$. For both the laboratory and the UMi scenario, more than 200 samples are collected in each subchannel to extract the statistical characteristics of tripolarized MIMO channels.

\section{Analysis and Modeling of Channel Properties}

Channel properties are highly related to the environment. This is because scatterers in environments determine the radio propagation mechanisms. In the following, we would analyze and model the channel properties of large-scale fading and small-scale fading in the laboratory and the UMi scenarios. The dependency of polarization directions on channel properties is also analyzed.

3.1. Path Loss. The path loss is normally caused by the natural expansion of the radio wavefront in free space, absorption losses, diffraction losses, and so on [16]. It is an important property in coverage prediction and interference analysis of radio communication systems [27-29]. Path loss can be usually modeled by [30]

$$
P L(\mathrm{~dB})=10 \log \frac{P_{T}}{P_{R}},
$$

where $P_{T}$ and $P_{R}$ are the transmitted power and the received power, respectively. The received power can be expressed as a function of distance, also known as Friis' law [16]:

$$
P_{R}(d)=P_{T} G_{T} G_{R}\left(\frac{\lambda}{4 \pi d}\right)^{2},
$$

where $G_{T}$ and $G_{R}$ are the antenna gain at the TX side and the RX side, respectively, $d$ is the spatial distance between the TX and the RX, and $\lambda$ is the wavelength. Free space is an ideal propagation environment, in which there is no obstruction. And, the free space path loss (FSPL) can be expressed as [31]

$$
P L_{F S P L}(f, d)=20 \log _{10}\left(\frac{4 \pi \mathrm{d} f}{c}\right),
$$

where $f$ is the frequency of a radio wave and $c$ is the speed of light.

In the realistic environment, scatterers distribute in the propagation environment and result in obstruction. This makes the FSPL model not available. To characterize the path loss in the real environment, we use the floating-intercept (FI) model, a popular used model in channel modeling standardization, to describe the path loss based on measured data [32-34]. The FI model can be expressed as 


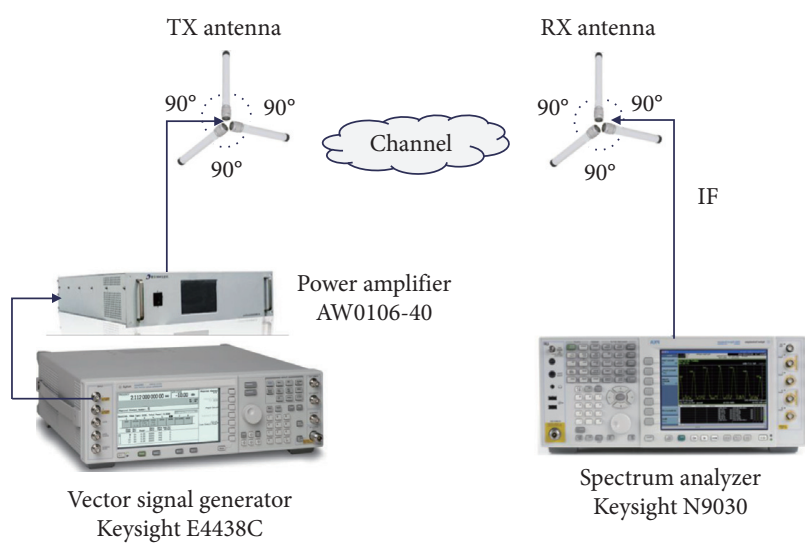

FIgURE 1: The structure of the used channel sounder.

$$
P L_{\mathrm{FI}}(d)[\mathrm{dB}]=\beta+10 \alpha \log _{10} d+X_{\sigma}^{\mathrm{FI}},
$$

where $\alpha$ is the slope of the model, $\beta$ is the floating-intercept, and $X_{\sigma}^{\mathrm{FI}}$ is a zero-mean Gaussian variable with a standard derivation $\sigma$ representing the shadowing. After removing the system response from measured data, the measured path loss in the channel between the $i$ th TX antenna and the $j$ th RX antenna can be obtained by

$$
P L_{i, j}=\sum_{n}^{N}\left|h_{i, j}\left(\tau_{n}\right)\right|^{2}, \quad n=1,2, \ldots, N,
$$

where $i$ and $j$ are the index of antenna positions, see Figure 2, $h_{i, j}$ is the channel impulse response between the $i$ th TX antenna and the $j$ th $\mathrm{RX}$ antenna, $\tau_{n}$ is the delay value in the $n$th delay bin, and $N$ is the number of delay bins. It is worth noting that system calibration and denoising should be done before obtaining the channel impulse response $[35,36]$.

Figure 7 shows fitting results of measured path loss by using the FI model (to make the figure look clear, measured path loss results are not plotted). The FSPL is also plotted to do a comparison. We can see that, at the same measured position, path loss varies as the polarization direction changes. Generally, the path loss is smaller for co-polarization (TX2-RX2 and TX3-RX3) channels than that for cross-polarization channels. For example, in Figure $7(\mathrm{a})$, the fitting lines of the path loss for co-polarization (TX2-RX2 and TX3-RX3) channels are at the bottom of the figure. This indicates that the power attenuation is severer in the crosspolarization channels. It is worth noting that the path loss for the TX1-RX co-polarization channel is specially large because the radio propagation is vertical to the TX-RX direction. Also, the slope of the FI model depends on the polarization combination. This indicates that power decays differently with the distance in different polarization directions due to polarization coupling and rotation. For example, in Figure $7(\mathrm{a})$, the path loss for 9 polarization combinations changes in the range of 47.34 to $57.65 \mathrm{~dB}$ at a distance of $2 \mathrm{~m}$. Also, the path loss for the TX 1 and the RX 1 combination is severer than other two co-polarization combinations. This is because the direction with the maximum gain of the dipole is perpendicular to the propagation direction, see Figure 3. In [24], it also shows that the slope of the path loss model for the vertically polarized subchannel is flatter than that for the horizontally polarized subchannel. Furthermore, as the propagation distance increases, path loss for some cross-polarization combinations increases slowly, like that for the TX 3 and the RX 1 combination. This is mainly because scattering in the propagation becomes richer due to extended space between the TX and the RX. And, this results in stronger polarization rotation and high cross-polarization coupling. In [24], we can also find that the slope becomes flatter for the vertical-to-horizontal subchannel. However, it still indicates that the path loss increases with the distance. This discrepancy may come from the environment difference. In our measured environment, the polarization rotation is stronger.

Table 2 lists fitting parameters of the FI model in different measured scenarios. In the laboratory, all slope values of the FI model are smaller than 2 under the LOS condition, while it is 2 in the free space. This can be explained by that the laboratory is a closed space, and the RX can receive more reflected multipaths (MPCs) from the floor, walls, and ceiling [37]. Under the NLOS condition, we can see that only the slope value for $P L_{2,1}$ is less than 2. Furthermore, the slope value for $P L_{3,3}$ is 5.58 , indicating high dependency on the distance. The ITU path loss model for the indoor office scenario shows a similar trend. For example, the slope value is 1.69 under the LOS condition, while it is 4.33 for the NLOS condition [33]. In the UMi scenario, the slope values of the FI model have large variances for both LOS and NLOS conditions. For instance, under the LOS condition, the slope value for $P L_{1,1}$ is 2.43 , while it is 1.29 for $P L_{3,3}$; under the NLOS condition, the slope value changes from 4.95 for $P L_{2,2}$ to 0.40 for $P L_{1,1}$. This mainly because that the UMi scenario is full of different kinds of scatterers, e.g., plants, buildings, cars, and humans, see Figure 5, which leads to a richscattering environment [38]. Also, polarization alignment is not easy to realize due to the large height difference between the TX and the RX. Basically, we can conclude that the path loss characteristic shows high dependency on the polarization combinations. However, the traditional ITU path loss model does not consider the effects of polarization combinations. Though XPD is introduced in generating smallscale fading response, it only characterizes the received power difference between co-polarization directions and cross-polarization directions, without considering that between co-polarization directions.

3.2. Delay Spread. DS describes the time dispersion of MPCs and can be used to calculate the coherence bandwidth $[37,39]$. Furthermore, large DS may cause intersymbol interference. Thus, it is essential to characterize the DS to improve system reliability. In a polarized MIMO system, delay dispersion reflects the degree of scattering and is related to polarization coupling and rotation. However, in current ITU channel models, the relationship between the DS and polarization is not considered [33]. Here, we study the statistics of the DS for different polarization combinations. The root mean square (RMS) DS in the channel 


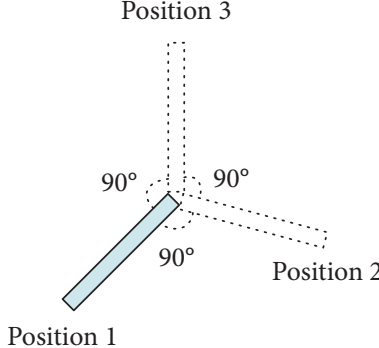

(a)

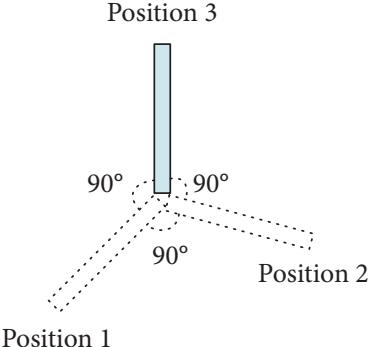

(b)

FIgURE 2: Tripolarized antenna rotating positions. (a) TX. (b) RX.

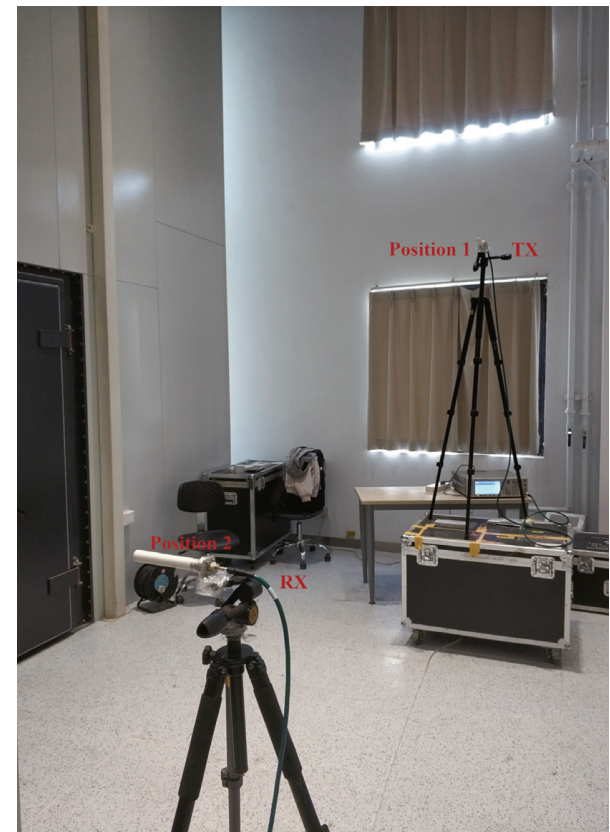

Figure 3: The picture of measurements in the laboratory.

between the ith TX antenna and the $j$ th RX antenna can be calculated as follows [40]:

$$
\sigma_{\tau_{i, j}}=\sqrt{\overline{\tau_{i, j}^{2}}-\bar{\tau}_{i, j}^{2}}
$$

where

$$
\begin{gathered}
\overline{\tau_{i, j}^{2}}=\frac{\sum_{n=0}^{N}\left|h_{i, j}\left(\tau_{n}\right)\right|^{2} \tau_{n}^{2}}{\sum_{n=0}^{N}\left|h_{i, j}\left(\tau_{n}\right)\right|^{2}}, \\
\bar{\tau}_{i, j}=\frac{\sum_{n=0}^{N}\left|h_{i, j}\left(\tau_{n}\right)\right|^{2} \tau_{n}}{\sum_{n=0}^{N}\left|h_{i, j}\left(\tau_{n}\right)\right|^{2}},
\end{gathered}
$$

where $\overline{\tau_{i, j}^{2}}$ and $\bar{\tau}_{i, j}$ are the second origin comment and the first origin comment of the delay spread in the channel between the $i$ th TX antenna and the $j$ th RX antenna, respectively.

Figure 8 describes the cumulative distribution function (CDF) of RMS DS in the laboratory. Basically, we can see
TABle 1: Measurement system parameters.

\begin{tabular}{lc}
\hline Parameter & Value \\
\hline Center frequency in the indoor/outdoor scenario & $3.5 / 4.9 \mathrm{GHz}$ \\
Symbol rate & $50 \mathrm{Mbaud} / \mathrm{s}$ \\
RF bandwidth & $100 \mathrm{MHz}$ \\
Transmitted power & $0 \mathrm{dBm}$ \\
Power amplifier gain & $43 \mathrm{~dB}$ \\
Dipole antenna gain & $2.15 \mathrm{~dB}$ \\
\hline
\end{tabular}

that RMS DS under the NLOS condition is larger than that under the LOS condition. Also, RMS DS in the UMi scenario is larger than that in the laboratory. This is in line with the forecast because MPCs are more likely to experience scattering and propagate longer distances in a more complex and larger environment [41]. Correspondingly, the degree of delay dispersion in the channel increases. Furthermore, it can be investigated that RMS DS changes between different polarization combinations. Especially, we can find a trend from Figure 8(a) that RMS DS in the channel for three copolarization combinations is smaller than that for other cross-polarization combinations. This is because the radio propagation is not the same as the TX-RX direction, and there are more reflected or scattered paths. In ITU channel models, RMS DS is modeled as a function of frequency above $6 \mathrm{GHz}$, while statistics of RMS DS are set to constants below $6 \mathrm{GHz}$ [33]. However, in realistic propagation environments, the radio wave with different polarization directions propagates in different routes. Also, as discussed in Section 3.2, the propagation loss is dependent on the polarization directions. Thus, according to equations (6) to (8), the RMS DS also has dependency on the polarization directions.

According to experimental investigations and ITU channel models, RMS DS can be modeled by a lognormal function [33, 42]. As shown in Figure 9, the lognormal fitting line fits well with the measured RMS DS in the UMi scenario under the NLOS condition. In other measurement cases, the lognormal fitting also works well. However, due to limited space, we do not plot all of them here but give a table of fitting parameters for 9 polarization combinations, see Table 3. In the laboratory, the mean value of the lognormal fitting under the LOS condition ranges from $26.37 \mathrm{~ns}$ for $\sigma_{\tau_{3,3}}$ to $36.40 \mathrm{~ns}$ for $\sigma_{\tau_{3,1}}$. It seems that there is a trend that the channel for copolarization combinations has smaller DS. Similar trend 


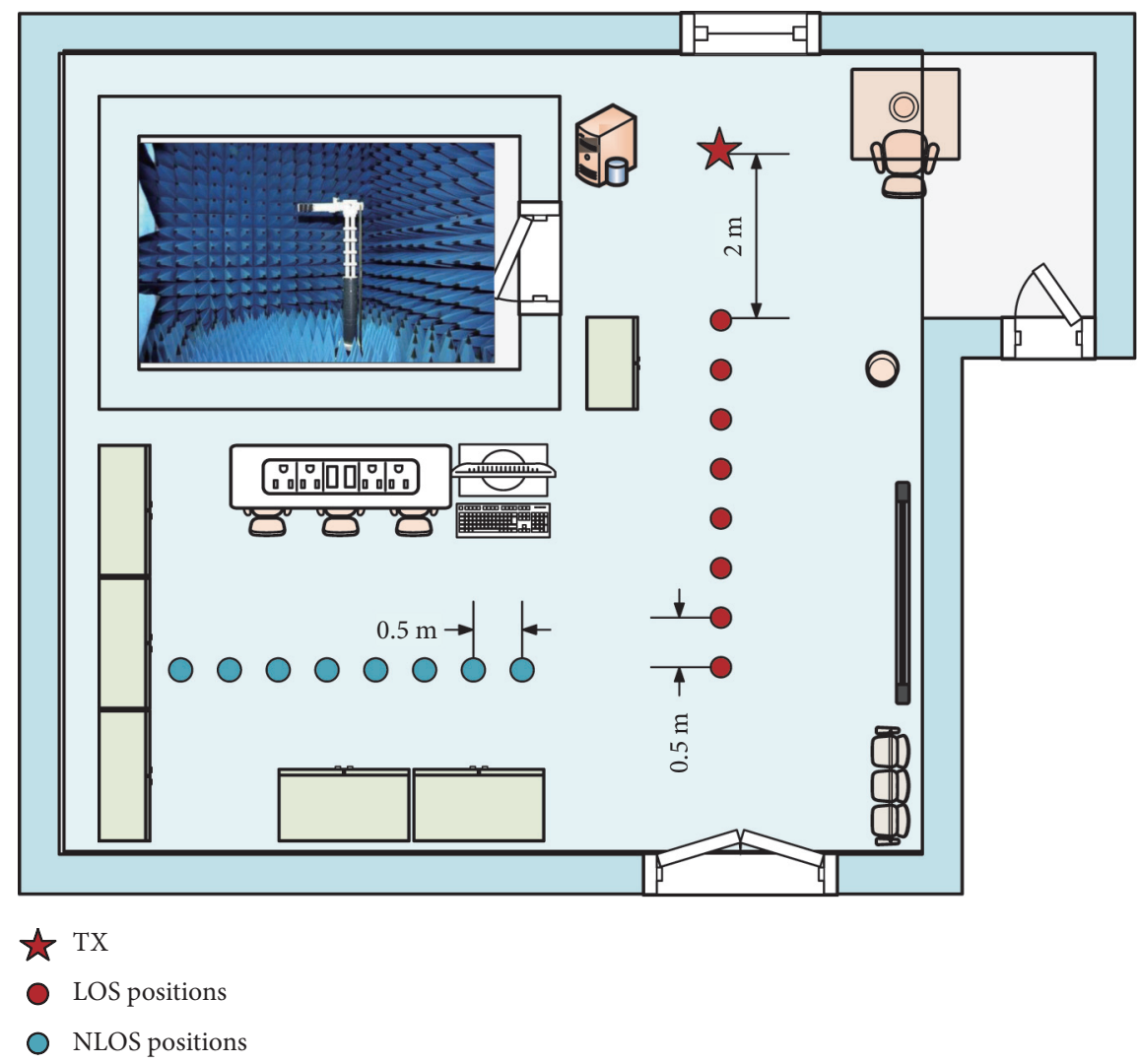

FIgURE 4: The sketch map of measurement positions in the laboratory.

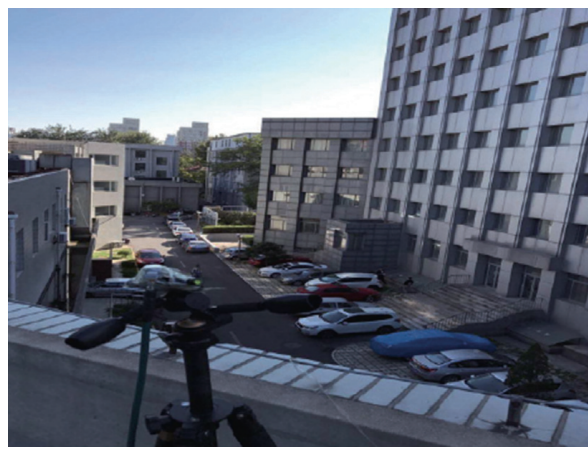

(a)

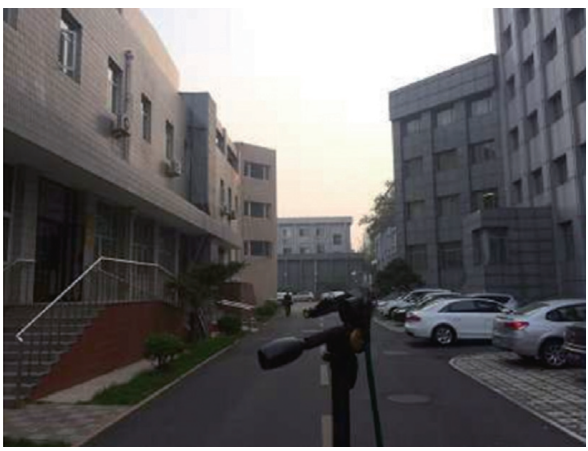

(b)

Figure 5: The views of the (a) TX antenna and (b) RX antenna.

can be observed under the NLOS condition. For example, the mean value of the lognormal fitting is $42.85 \mathrm{~ns}$ for $\sigma_{\tau_{2},}$, while it is $55.16 \mathrm{~ns}$ for $\sigma_{\tau_{23}}$, increasing about $12.31 \mathrm{~ns}$. In the UMi scenario, the RMS DS is almost triple compared to that in the laboratory due to more scattering paths. Also, the co-polarization channel for the TX 2 and the RX 2 gets the smallest mean value, i.e., 58.77 ns under the LOS condition and 111.98 ns under the NLOS condition. This can be explained by Figure 10, which shows an example of power delay profiles (PDPs) in the channel of $h_{1,1}$ and $h_{2,2}$ in the UMi scenario under the LOS condition. We can see that though those two PDPs are similar, the LOS path has stronger power in the channel of $h_{1,2}$, while there are less reflected MPCs around the LOS path. This results in weaker delay dispersion and smaller DS. According to authors' knowledge, there is no literature that gives an investigation on the delay characteristics in the tripolarized MIMO channels. However, we can find supporting results in [43] that the delay dispersion becomes stronger in a larger space or under the NLOS condition.

3.3. Cross-Polarization Discrimination. In the propagation, the polarization orientation of electromagnetic waves will change after reflection and scattering. This process is called 


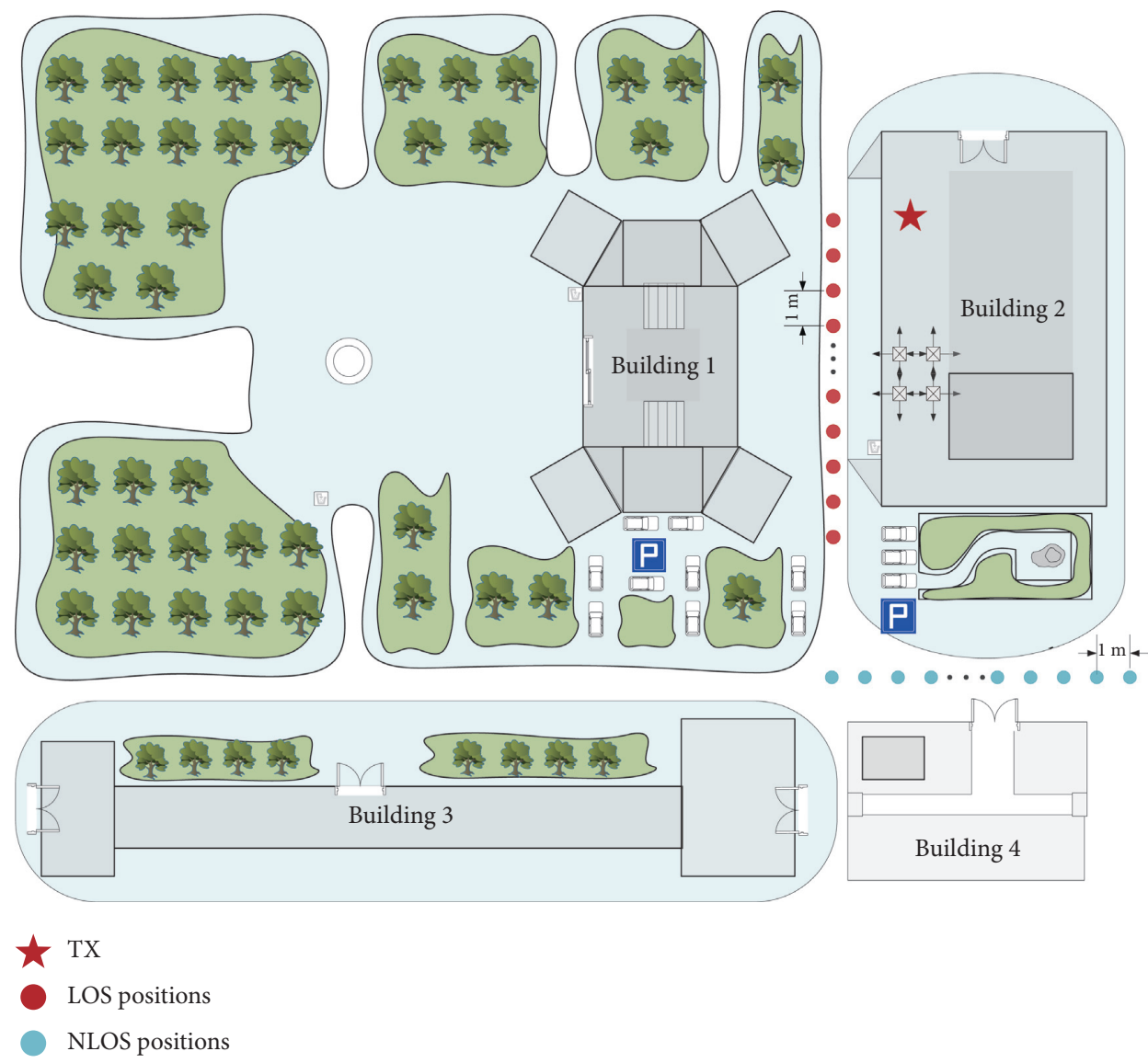

FIGURE 6: The sketch map of measurement positions in the UMi scenario.

channel depolarization and can result in power imbalance [44]. As analyzed in Section 3.2, the path loss changes in different polarized channels. XPD is used to characterize this process, which is defined as the power ratio of different polarization orientations. Also, it denotes the amount of power leakage from one polarization to another. According to that definition, XPD for cross-polarization combinations can be written as

$$
\mathrm{XPD}_{x x / x y}(\mathrm{~dB})=10 \log _{10}\left(\frac{\varepsilon\left\{\left|h_{x x}\right|^{2}\right\}}{\varepsilon\left\{\left|h_{x y}\right|^{2}\right\}}\right), \quad x \neq y,
$$

where $\left|h_{x x}\right|^{2}$ indicates the channel gain from the TX $x$ to RX $x,\left|h_{x y}\right|^{2}$ indicates the channel gain from the TX $x$ to the RX $y$, and $\varepsilon\{\cdot\}$ is the expectation operator. To fully characterize the polarimetric behavior of the channel, other XPDs for copolarization combinations are also needed. They can be expressed by

$$
\mathrm{XPD}_{x x / y y}(\mathrm{~dB})=10 \log _{10}\left(\frac{\varepsilon\left\{\left|h_{x x}\right|^{2}\right\}}{\varepsilon\left\{\left|h_{y y}\right|^{2}\right\}}\right), \quad x \neq y .
$$

Figure 11 gives the probability density functions of XPD in $\mathrm{dB}$ for cross-polarization combinations in the laboratory and the UMi scenario. Also, XPD in $\mathrm{dB}$ is fitted with a normal function. It can be seen that, in the laboratory, the fitting parameters $\mu$ and $\sigma$ are $4.23 \mathrm{~dB}$ and $4.69 \mathrm{~dB}$, respectively, under the LOS condition, while they are $3.33 \mathrm{~dB}$ and $4.21 \mathrm{~dB}$, respectively, under the NLOS condition. It seems that power leakage is severer in the NLOS case. The same trend can be found in the UMi scenario, see Figure 11(b). Especially, the mean value under the LOS condition in the UMi scenario is $6.48 \mathrm{~dB}$, apparently larger than $1.68 \mathrm{~dB}$ under the NLOS condition. In [33], the mean value of XPD under the LOS condition is also larger than that under the NLOS condition for typical indoor and outdoor scenarios. This may be because, in the closed space, more radio waves experience polarization rotation after reflection, refraction, or diffusion. Also, due to smaller propagation distances, these radio waves have strong powers. Though the power leakage is severer in the laboratory, it is worth noting that the path loss in the laboratory is relatively smaller than that at the same propagation distance in the UMi scenario, see the analysis in Section 3.1.

To further investigate power leakage for all polarization combinations, Table 4 lists the fitting parameters of XPD for 6 cross-polarization combinations and 2 co-polarization combinations. A smaller mean value denotes that the polarization direction of radio waves is more likely to be rotated. Thus, it can be seen that polarization rotation is strong for the TX 1 and the RX 1 in both the laboratory and the UMi scenario. According to Figures 2 and 3, we can say that power leakage is more likely to happen in horizontal-to- 

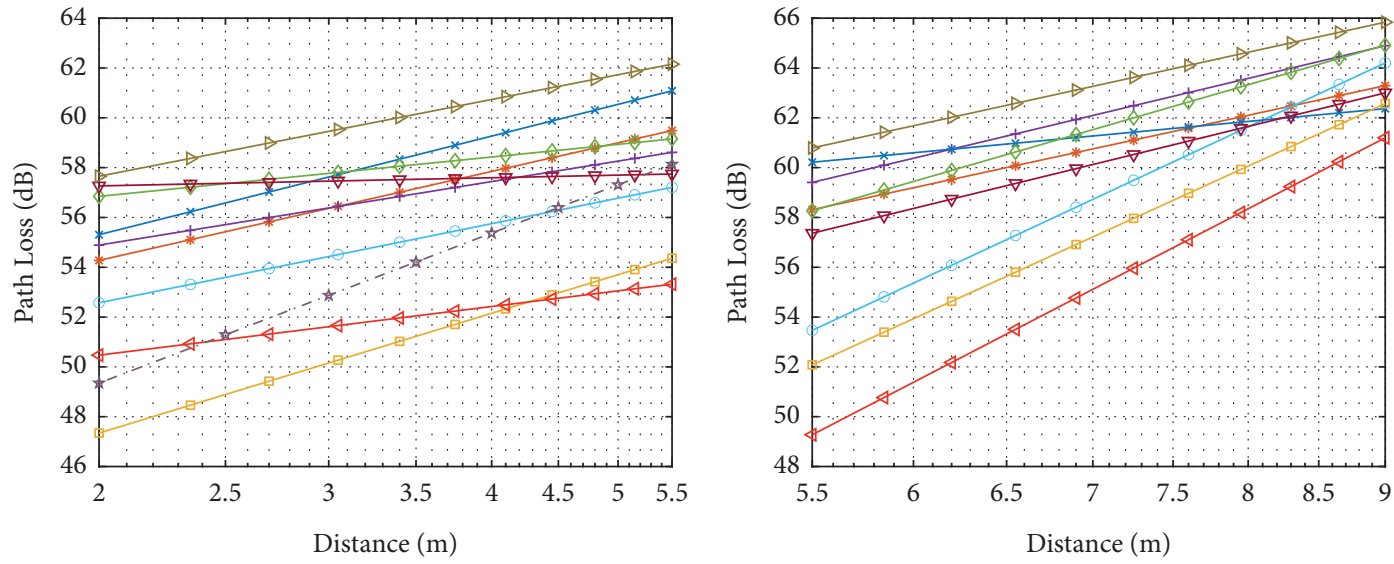

$$
\begin{array}{lll}
\rightarrow P L_{1,1} & \square P L_{2,2} & \rightarrow P L_{3,2} \\
\rightarrow P L_{1,2} & \multimap P L_{2,3} & \multimap P L_{3,3} \\
\multimap P L_{1,3} & \rightarrow P L_{3,1} & \rightarrow P L_{F S P L} \\
\text { × } P L_{2,1} & &
\end{array}
$$

(a)

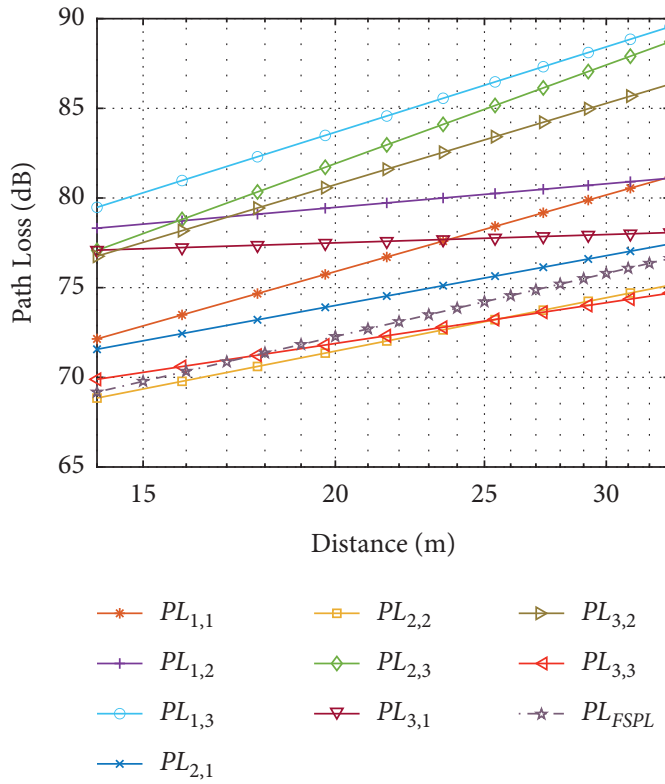

(c)

$$
\begin{array}{lll}
\rightarrow P L_{1,1} & \rightarrow P L_{2,1} & \rightarrow P L_{3,1} \\
\rightarrow P L_{1,2} & \rightarrow P L_{2,2} & \rightarrow P L_{3,2} \\
\multimap P L_{1,3} & \multimap P L_{2,3} & \hookrightarrow P L_{3,3}
\end{array}
$$

(b)

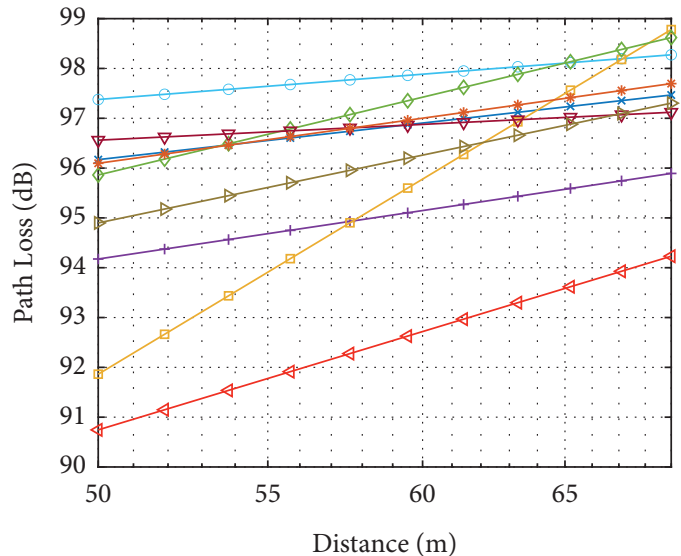

\begin{tabular}{|c|c|c|c|c|c|c|c|c|c|c|c|c|}
\hline \multirow{3}{*}{ Scenario } & \multicolumn{6}{|c|}{ Laboratory } & \multicolumn{6}{|c|}{ UMi } \\
\hline & \multicolumn{3}{|c|}{ LOS } & \multicolumn{3}{|c|}{ NLOS } & \multicolumn{3}{|c|}{ LOS } & \multicolumn{3}{|c|}{ NLOS } \\
\hline & $\alpha$ & $\beta$ & $\sigma$ & $\alpha$ & $\beta$ & $\sigma$ & $\alpha$ & $\beta$ & $\sigma$ & $\alpha$ & $\beta$ & $\sigma$ \\
\hline$P L_{1,1}(\mathrm{~dB})$ & 1.19 & 50.70 & 0.90 & 2.33 & 41.08 & 1.54 & 2.43 & 44.34 & 2.19 & 1.14 & 76.67 & 0.57 \\
\hline$P L_{1,2}(\mathrm{~dB})$ & 0.85 & 52.34 & 2.29 & 2.57 & 40.41 & 0.94 & 0.75 & 69.75 & 2.28 & 1.29 & 73.31 & 1.09 \\
\hline$P L_{1,3}(\mathrm{~dB})$ & 1.06 & 49.40 & 2.32 & 5.02 & 16.29 & 2.35 & 2.70 & 48.49 & 1.14 & 0.64 & 86.49 & 0.36 \\
\hline$P L_{2,1}(\mathrm{~dB})$ & 1.32 & 51.35 & 1.98 & 1.01 & 52.76 & 1.71 & 1.58 & 53.49 & 1.05 & 0.93 & 80.42 & 0.43 \\
\hline$P L_{2,2}^{2,1}(\mathrm{~dB})$ & 1.60 & 42.53 & 0.96 & 4.90 & 15.77 & 2.18 & 1.69 & 49.47 & 1.23 & 4.95 & 96.42 & 1.51 \\
\hline$P L_{2,3}(\mathrm{~dB})$ & 0.53 & 55.27 & 2.45 & 3.11 & 35.25 & 1.64 & 3.12 & 41.28 & 1.73 & 1.97 & 62.35 & 0.83 \\
\hline$P L_{3,1}(\mathrm{~dB})$ & 0.11 & 56.94 & 1.44 & 2.64 & 37.85 & 1.44 & 0.26 & 74.07 & 2.11 & 0.40 & 89.76 & 0.61 \\
\hline$P L_{3,2}(\mathrm{~dB})$ & 1.03 & 54.57 & 2.30 & 2.36 & 43.31 & 0.64 & 2.58 & 47.24 & 1.71 & 1.72 & 65.70 & 1.05 \\
\hline$P L_{3,3}(\mathrm{~dB})$ & 0.65 & 48.52 & 2.49 & 5.58 & 10.00 & 2.41 & 1.29 & 55.10 & 1.16 & 2.49 & 48.40 & 0.96 \\
\hline
\end{tabular}

$$
\begin{array}{lll}
\rightarrow P L_{1,1} & \rightarrow P L_{2,1} & \rightarrow P L_{3,1} \\
\rightarrow P L_{1,2} & \rightarrow P L_{2,2} & \rightarrow P L_{3,2} \\
\multimap P L_{1,3} & \multimap P L_{2,3} & \triangleleft P L_{3,3}
\end{array}
$$

(d)

FIgure 7: Path loss results in laboratory and UMi scenarios for different polarization combinations. (a) Path loss results under the LOS condition in the laboratory. (b) Path loss results under the NLOS condition in the laboratory. (c) Path loss results under the LOS condition in the UMi scenario. (d) Path loss results under the NLOS condition in the UMi scenario. (e) Legend.

TABle 2: Path loss fitting parameters of the FI model. 

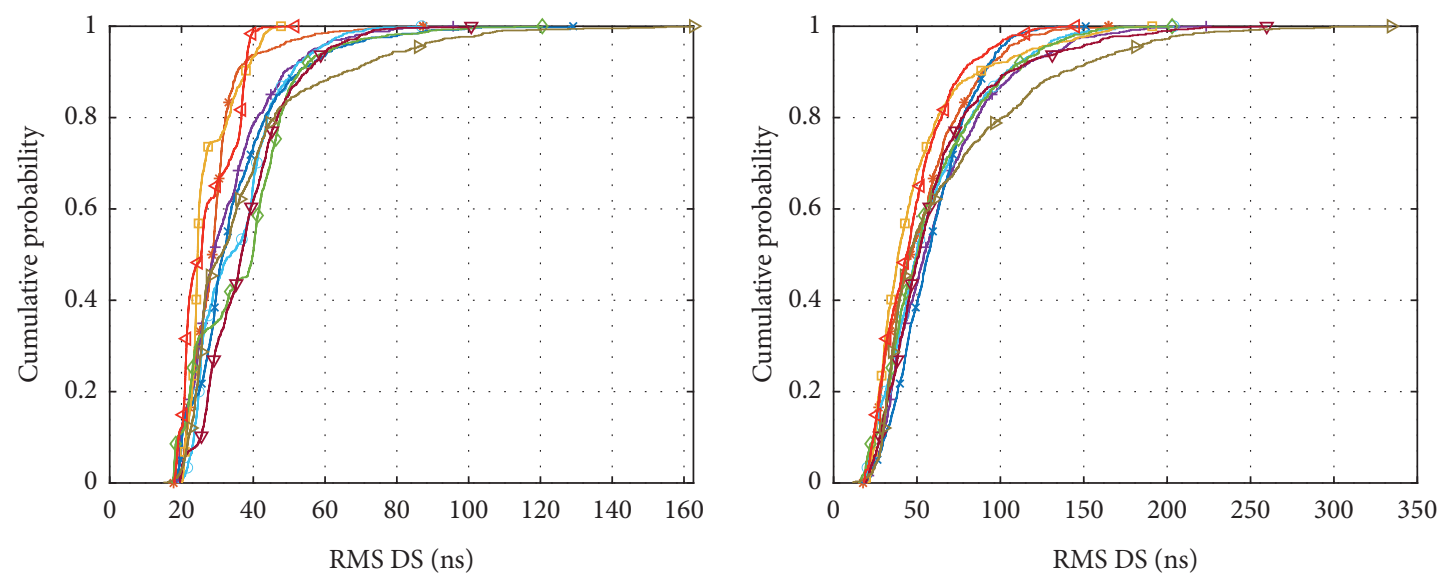

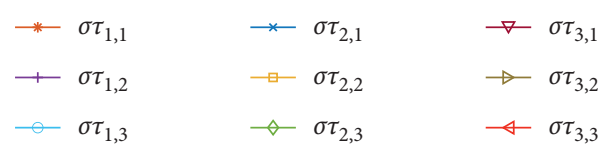

(a)

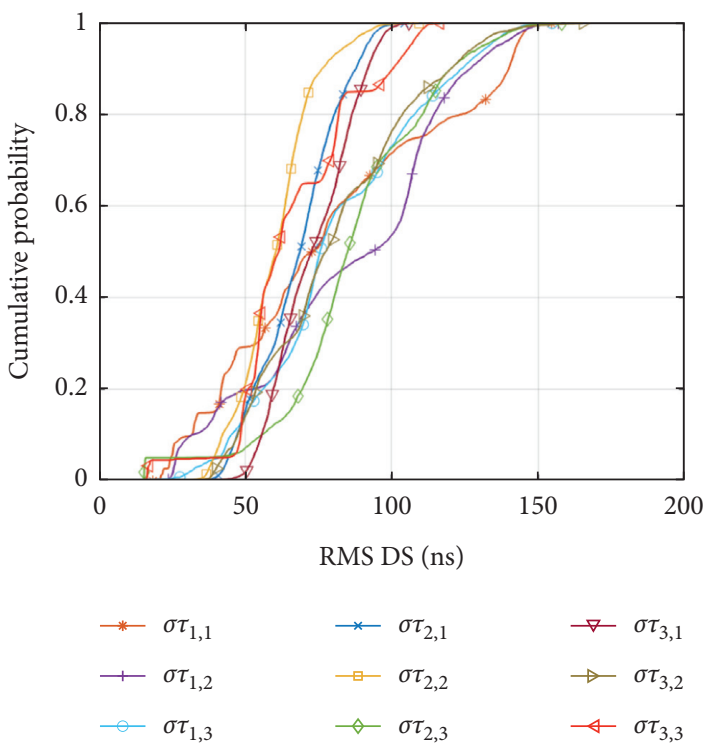

(c)

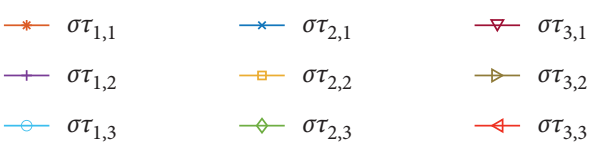

(b)
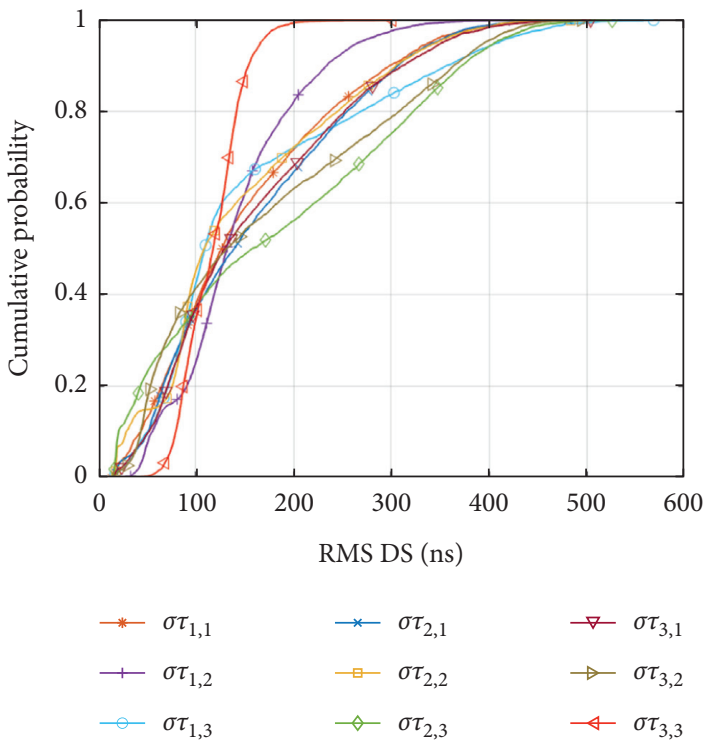

(d)

FIGURE 8: RMS DS results in laboratory and UMi scenarios for different polarization combinations. (a) RMS DS results under the LOS condition in the laboratory. (b) RMS DS results under the NLOS condition in the laboratory. (c) RMS DS results under the LOS condition in the UMi scenario. (d) RMS DS results under the NLOS condition in the UMi scenario. (e) Legend.

horizontal channels because the radio propagation direction may be different from the TX-RX direction. And, the propagation condition is better in vertical-to-vertical channels. Similar trends have been investigated in $[15,22]$. In [15], it is found that polarization rotation is strong when the dipole is parallel to the ground, but the polarization is maintained when it is vertical to the ground. Furthermore, this is consistent with the analysis in Section 3.1.

\section{Performance Evaluation of Tripolarized MIMO Systems}

Some efforts have been made to investigate the performance of tripolarized MIMO systems $[19,20]$. However, most of them do not consider realistic environments. In this section, we would give a performance evaluation of tripolarized MIMO systems based on channel measurements in realistic 


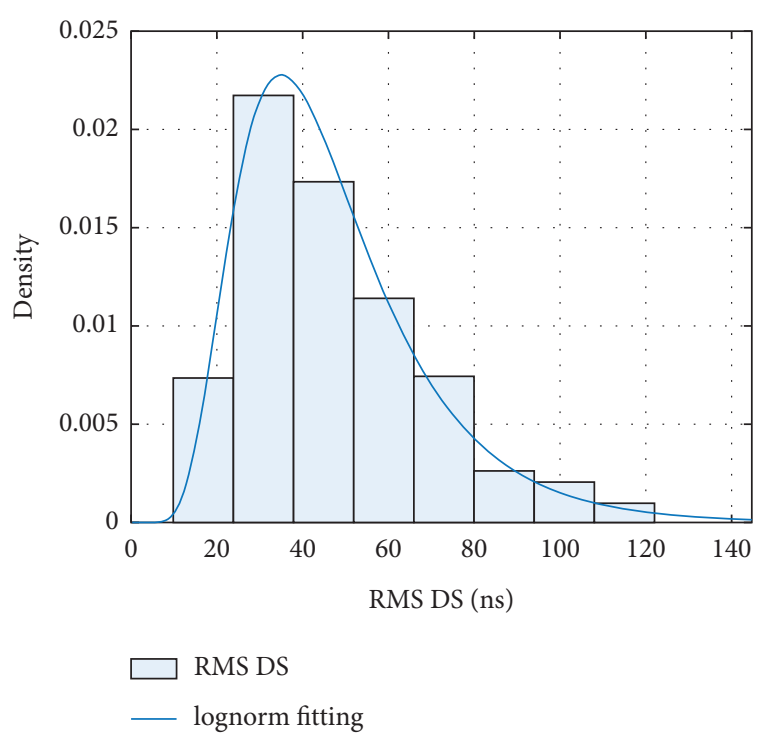

FIGURE 9: The RMS DS fitting results by using a lognormal model.

TABLE 3: RMS DS fitting parameters by using a lognormal model for 9 polarization combinations.

\begin{tabular}{|c|c|c|c|c|c|c|c|c|}
\hline \multirow{3}{*}{ Scenario } & \multicolumn{4}{|c|}{ Laboratory } & \multicolumn{4}{|c|}{$\mathrm{UMi}$} \\
\hline & \multicolumn{2}{|c|}{ LOS } & \multicolumn{2}{|c|}{ NLOS } & \multicolumn{2}{|c|}{ LOS } & \multicolumn{2}{|c|}{ NLOS } \\
\hline & $\mu$ & $\sigma$ & $\mu$ & $\sigma$ & $\mu$ & $\sigma$ & $\mu$ & $\sigma$ \\
\hline$\sigma_{\tau_{1,1}}(\mathrm{~ns})$ & 28.31 & 0.24 & 46.27 & 0.50 & 68.61 & 0.55 & 118.37 & 0.75 \\
\hline$\sigma_{\tau_{1,1}}^{i_{1,1}}(\mathrm{~ns})$ & 30.84 & 0.34 & 55.16 & 0.51 & 76.82 & 0.50 & 125.78 & 0.51 \\
\hline$\sigma_{\tau_{1,3}}(\mathrm{~ns})$ & 33.79 & 0.31 & 50.62 & 0.53 & 76.36 & 0.38 & 122.55 & 0.74 \\
\hline$\sigma_{\tau_{2,1}^{1,3}}(\mathrm{~ns})$ & 32.92 & 0.36 & 54.59 & 0.40 & 66.07 & 0.22 & 127.05 & 0.73 \\
\hline$\sigma_{\tau_{2,2}}^{2,1}$ (ns) & 26.46 & 0.21 & 42.85 & 0.49 & 58.77 & 0.22 & 111.98 & 0.81 \\
\hline$\sigma_{\tau_{2,3}}(\mathrm{~ns})$ & 34.63 & 0.40 & 50.38 & 0.53 & 79.85 & 0.45 & 123.16 & 1.01 \\
\hline$\sigma_{\tau_{3,1}^{2,3}}(\mathrm{~ns})$ & 36.40 & 0.31 & 52.62 & 0.51 & 71.88 & 0.19 & 127.21 & 0.71 \\
\hline$\sigma_{\tau_{3,2}}(\mathrm{~ns})$ & 34.10 & 0.43 & 54.96 & 0.62 & 76.16 & 0.34 & 126.92 & 0.83 \\
\hline$\sigma_{\tau_{3,3}}(\mathrm{~ns})$ & 26.37 & 0.26 & 43.00 & 0.45 & 62.21 & 0.38 & 112.80 & 0.27 \\
\hline
\end{tabular}

measured scenarios, which can give a better insight into the application of tripolarized MIMO systems.

4.1. Demmel Condition Number. The Demmel condition number is a useful indicator to characterize the spatial multiplexing performance in channels [45]. Also, if the Demmel condition number is below a certain threshold, it is better to use spatial multiplexing rather than diversity transmission. The threshold is related to the discrepancy between the space-time codewords of the diversity scheme or the different received space-time codewords [46]. The Demmel condition number is defined in [47] as

$$
K_{D}=\|\mathbf{H}\|_{\mathrm{F}}\left\|\mathbf{H}^{-1}\right\|_{2}=\frac{\|\mathbf{H}\|_{\mathrm{F}}}{\sqrt{\lambda_{\min }(\mathbf{H})}}
$$

where $\mathbf{H}$ is the $3 \times 3$ MIMO channel matrix consisted of measured channel impulse responses for 9 polarization combinations, $\|\cdot\|_{\mathrm{F}}$ is the Frobenius norm, and $\lambda_{\min }(\mathbf{H})$ is the smallest singular value of $\mathbf{H}$.
Figure 12 shows the CDFs of $K_{D}$ in the laboratory and the UMi scenario. Generally, the distribution of $K_{D}$ is similar in these two scenarios. $K_{D}$ ranges from around $2.42 \mathrm{~dB}$ to around $49.76 \mathrm{~dB}$. In [22], it is found that $K_{D}$ for the tripolarized MIMO system ranges from around $8 \mathrm{~dB}$ to around $40 \mathrm{~dB}$. Furthermore, we can see that the mean value of $K_{D}$ under the LOS condition is relatively larger than that under the LOS condition in the laboratory. Thus, the LOS case may be more suitable for diversity transmission. However, the opposite phenomenon is observed in the UMi scenario. It seems that the LOS case is more suitable for diversity transmission. It is worth noting that the discrepancy of $K_{D}$ between different cases is not large. The trend may be different in other measurements, but the exact values of $k_{D}$ can be an indicator of using spatial multiplexing or diversity transmission.

4.2. Capacity Gain. The channel capacity reflects the maximum amount of information that can be transmitted by the channel, which is a popular indicator of judging the performance of wireless communication systems [48]. Channel correlation indicates the similarity between subchannels and limits the achievable capacity of MIMO systems [49]. According to its definition, the correlation coefficient can be obtained by

$$
\rho=\left|\frac{\varepsilon\left[h_{x, y} h_{z, w}^{*}\right]-\varepsilon\left[h_{x, y}\right] \varepsilon\left[h_{z, w}^{*}\right]}{\sqrt{\left(\varepsilon\left[\left|h_{x, y}\right|^{2}\right]-\left|\varepsilon\left[h_{x, y}\right]\right|^{2}\right)\left(\varepsilon\left[\left|h_{z, w}\right|^{2}\right]-\left|\varepsilon\left[h_{z, w}\right]\right|^{2}\right)}}\right|,
$$

where $*$ is the complex conjugate operation, $|\cdot|$ is the modulus of complex value, and $h_{z, w}$ is the channel impulse response between the $z$ th TX antenna and the $w$ th $\mathrm{RX}$ antenna. Figure 13 gives the correlation coefficient results under the LOS condition in the UMi scenario. It can be observed that the correlation coefficient between different polarized channels is generally small. Furthermore, the correlation coefficient ranges from 0.02 to 0.16 . This indicates that subchannels in tripolarized MIMO systems have low correlation and corresponding leads to a large channel capacity.

To further evaluate the performance of tripolarized MIMO systems, we compare the channel capacity gain of tripolarized MIMO systems with that of single-polarized MIMO systems. The channel capacity is defined as [9]

$$
C=\frac{1}{B} \int_{B} \log _{2}\left\{\operatorname{det}\left(I_{N_{t}}+\frac{\rho}{N_{t}} \mathbf{H}(f) \mathbf{H}^{\mathrm{H}}(f)\right)\right\} \mathrm{d} f,
$$

where $\rho$ is the received SNR, $B$ is the channel bandwidth, $N_{t}$ is the number of antenna elements at the TX side, $(\cdot)^{\mathrm{H}}$ stands for matrix conjugate transposition, and $\mathbf{H}(f)$ is the normalized channel frequency response as $\varepsilon\left\{\|\mathbf{H}(f)\|_{F}^{2}\right\}=N_{t} \times N_{r}$ (the normalization is to remove the effects of large scale fading so that the impacts of the MPC richness can be better illustrated), where $N_{r}$ is the number of antenna elements at the RX side. In this paper, $N_{t}$ and $N_{r}$ are both equal to 3 . Furthermore, the vertical-to-vertical unipolarized subchannel capacity (based on 


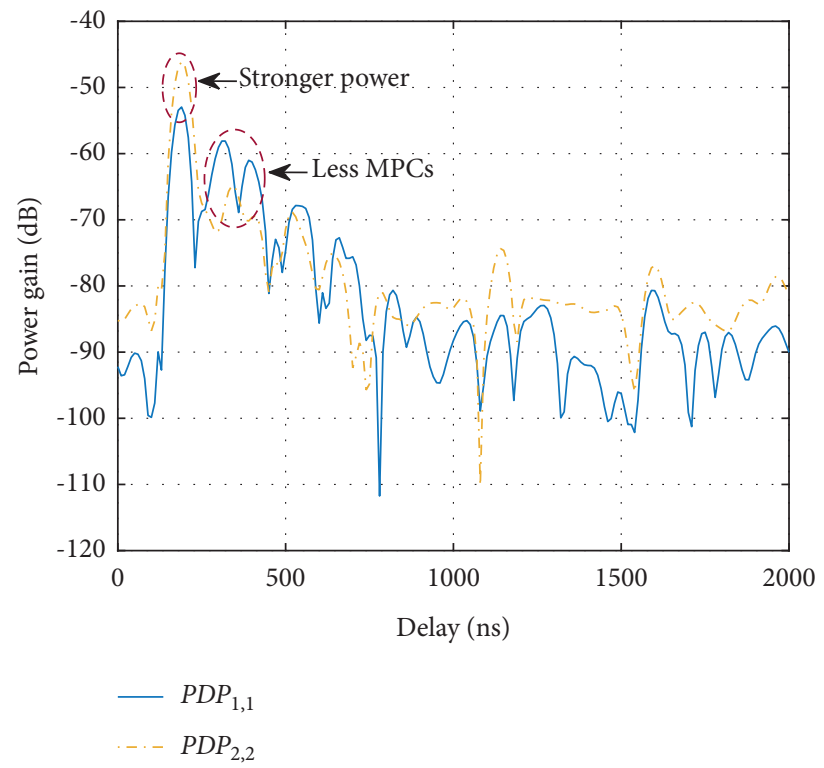

Figure 10: The PDP comparison between the subchannel $h_{1,1}$ and the subchannel $h_{2,2}$.

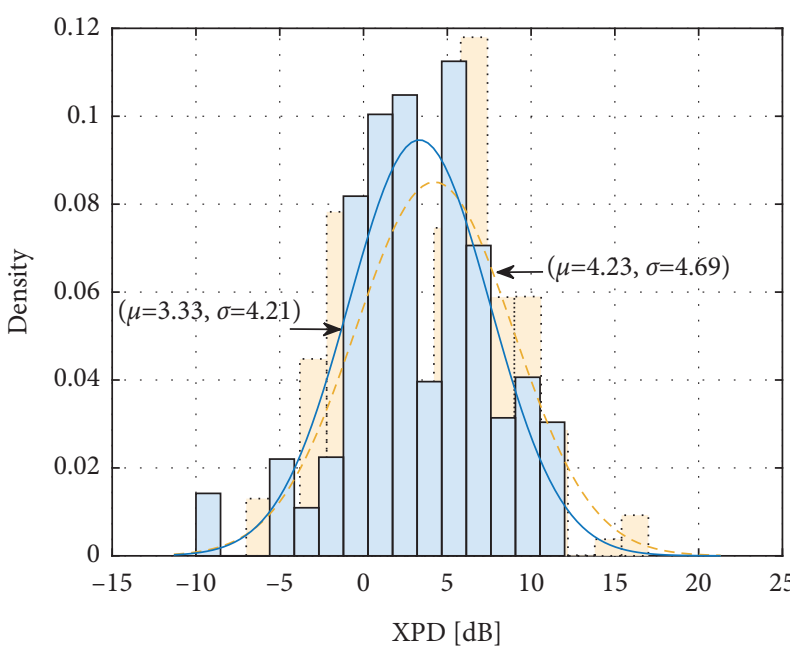

LOS

NLOS
_ _ _ Normal fitting-LOS

— Normal fitting-NLOS

(a)

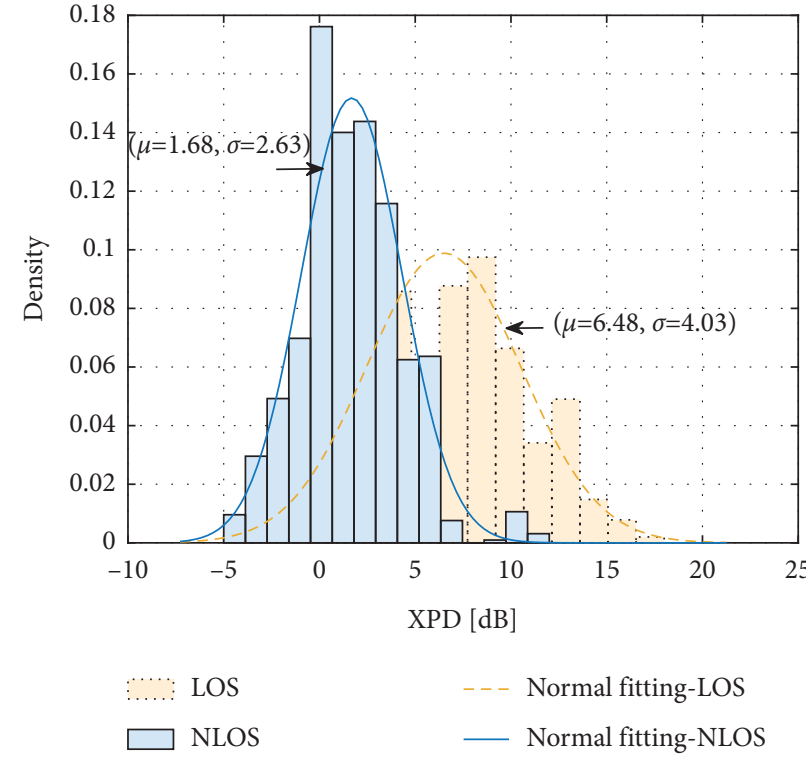

(b)

FIgURE 11: The probability density functions of XPD in laboratory and UMi scenarios. (a) The probability density functions of XPD in the laboratory. (b) The probability density functions of XPD in the UMi scenario.

the aforementioned analysis, the propagation condition in the channel for the TX 3 and RX 3 is generally better than that for other polarization combinations; thus, the vertical-to-vertical unipolarized subchannel capacity can be a better reference to demonstrate the performance of tripolarized MIMO systems) is selected as the referenced baseline and the gains of tripolarized channels are calculated as

$$
K_{G}=\frac{C_{\text {tri }}}{C_{\text {uni }}}
$$

where $C_{\text {tri }}$ indicates the capacity of the tripolarized MIMO channel and $C_{\text {uni }}$ is the referenced vertical-to-vertical unipolarized channel capacity.

Figure 14 shows the cumulative probability of capacity in laboratory and UMi scenarios. Both the tripolarized channel capacity $C_{u n i}$ and the vertical-to-vertical unipolarized subchannel capacity $C_{\text {uni }}$ are plotted in the figure. We can see that $C_{\text {tri }}$ is apparently larger than $C_{\text {uni }}$. For example, $C_{\text {tri }}$ is $16.81 \mathrm{bps} / \mathrm{Hz}$ at the cumulative probability of $50 \%$, while $C_{\text {uni }}$ is $6.58 \mathrm{bps} / \mathrm{Hz}$ under the LOS condition in the laboratory. Furthermore, the channel capacity under the NLOS 
TABLE 4: The statistical results of XPD for 6 cross-polarization combinations and 2 co-polarization combinations.

\begin{tabular}{|c|c|c|c|c|c|c|c|c|}
\hline \multirow{3}{*}{ Scenario } & \multicolumn{4}{|c|}{ Laboratory } & \multicolumn{4}{|c|}{ UMi } \\
\hline & \multicolumn{2}{|c|}{ LOS } & \multicolumn{2}{|c|}{ NLOS } & \multicolumn{2}{|c|}{ LOS } & \multicolumn{2}{|c|}{ NLOS } \\
\hline & $\mu$ & $\sigma$ & $\mu$ & $\sigma$ & $\mu$ & $\sigma$ & $\mu$ & $\sigma$ \\
\hline $\mathrm{XPD}_{11 / 12}(\mathrm{~dB})$ & -0.24 & 1.58 & 1.37 & 1.55 & 2.64 & 2.66 & -1.86 & 1.41 \\
\hline $\mathrm{XPD}_{11 / 13}(\mathrm{~dB})$ & -2.03 & 2.45 & -1.77 & 3.82 & 7.93 & 2.57 & 0.91 & 0.90 \\
\hline $\mathrm{XPD}_{22 / 21}(\mathrm{~dB})$ & 7.26 & 1.95 & 3.68 & 4.30 & 2.48 & 1.35 & 1.35 & 2.43 \\
\hline $\mathrm{XPD}_{22 / 23}(\mathrm{~dB})$ & 6.81 & 3.11 & 4.13 & 2.70 & 11.26 & 2.76 & 1.81 & 2.41 \\
\hline $\mathrm{XPD}_{33 / 31}(\mathrm{~dB})$ & 5.44 & 3.04 & 4.73 & 2.95 & 5.03 & 2.25 & 4.28 & 1.43 \\
\hline $\mathrm{XPD}_{33 / 32}(\mathrm{~dB})$ & 8.12 & 3.82 & 7.84 & 3.12 & 9.58 & 1.82 & 3.59 & 1.22 \\
\hline $\mathrm{XPD}_{11 / 22}(\mathrm{~dB})$ & -5.90 & 1.65 & -3.29 & 3.12 & -4.85 & 2.83 & -1.44 & 3.00 \\
\hline $\mathrm{XPD}_{22 / 33}(\mathrm{~dB})$ & 0.75 & 2.43 & -2.04 & 2.86 & -4.65 & 2.18 & -4.36 & 1.97 \\
\hline
\end{tabular}

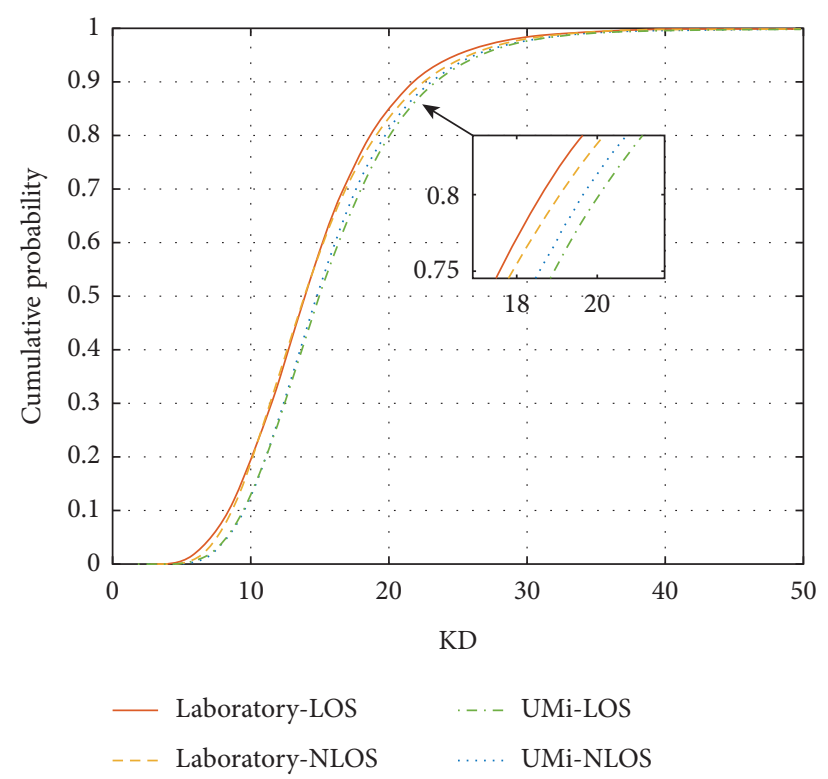

FIgure 12: The CDFs of $K_{D}$ in the laboratory and the UMi scenario.

condition is larger than that under the LOS condition because the degree of freedom is larger under the NLOS condition. This is within the expectation, and similar results can be found in $[9,50]$. In the following, we would quantitatively analyze the channel capacity gain.

Figure 15 gives the CDFs of $K_{G}$ in the laboratory and the UMi scenario. Generally, the obtained channel capacity of tripolarized MIMO systems in these two scenarios is quite similar. Furthermore, though the polarization rotation happens and is strong for some polarization combinations as discussed in the above manuscript, we can see that the channel capacity gain is still considerable. This is consistent with analysis results in [15]. Table 5 lists the channel capacity gain values in the laboratory and the UMi scenario by giving a special cumulative probability. We can see that only $10 \%$ channel capacity gains are less than 2.08. Thus, even though the polarization rotation happens, the channel capacity of tripolarized MIMO systems can become 2 times of the unipolarized case. Also, 50\% channel capacity gains are less than 2.48 , and $90 \%$ of channel capacity gains are less than 2.81, close to 3. In [15], the mean value of $K_{G}$ is about 2.50. This indicates that 3 independent subchannels can be supported by tripolarized MIMO systems in both the laboratory and the UMi scenario. 


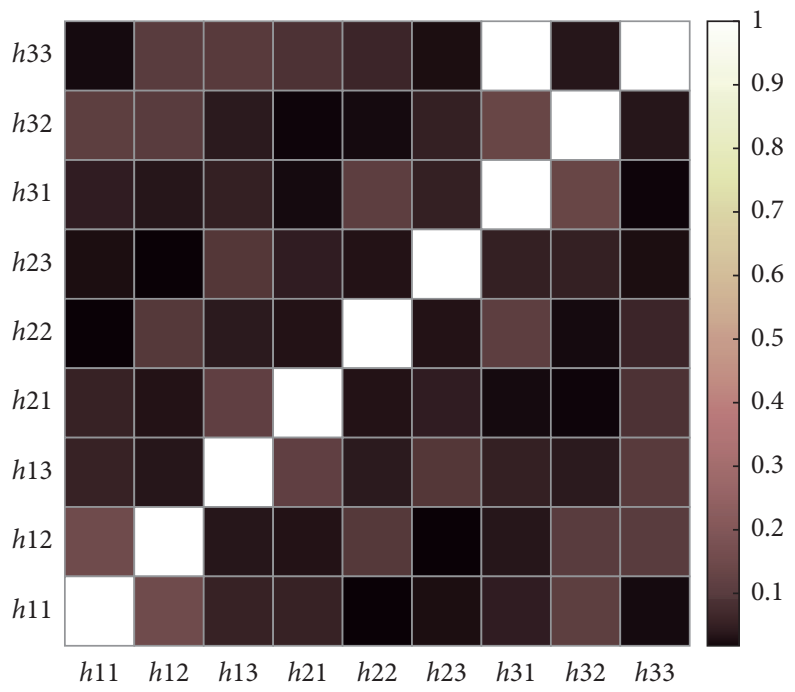

FIgURE 13: The CDFs of $K_{D}$ in the laboratory and the UMi scenario.

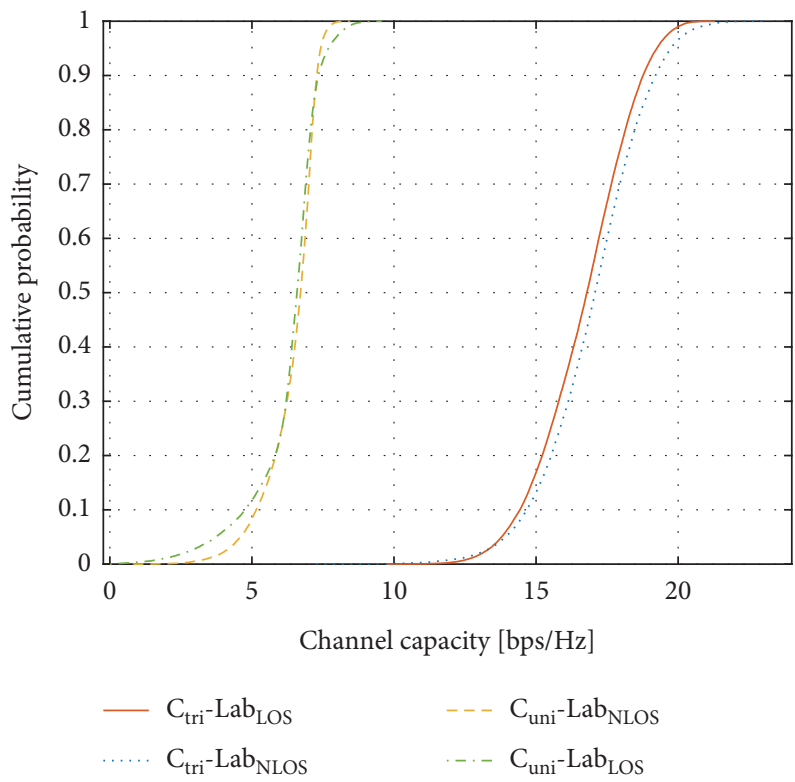

(a)

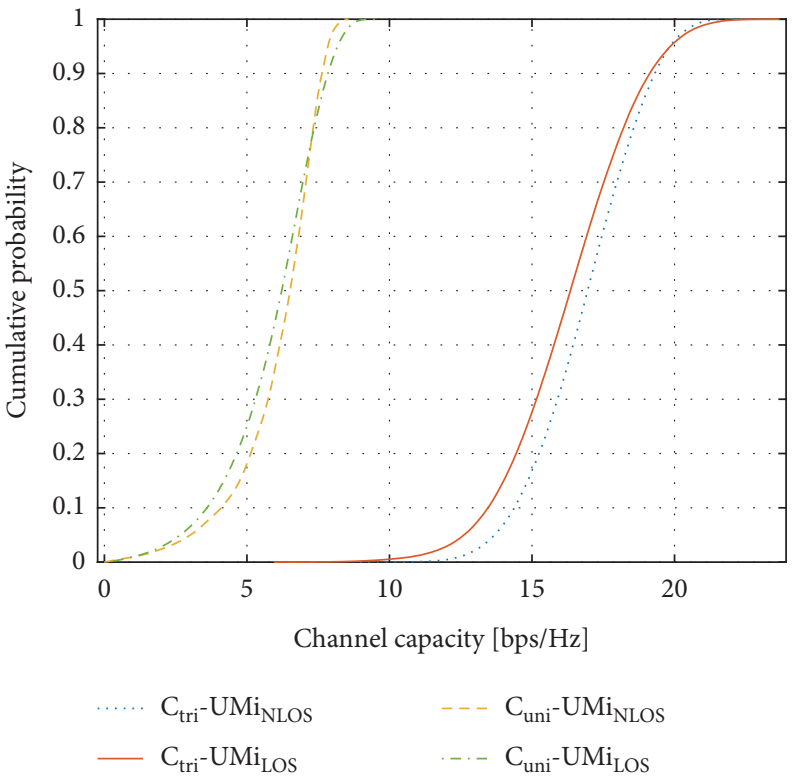

(b)

FIGURE 14: The cumulative probability of capacity in laboratory and UMi scenarios. $\rho$ is set to $20 \mathrm{~dB}$. (a) Laboratory. (b) UMi. 


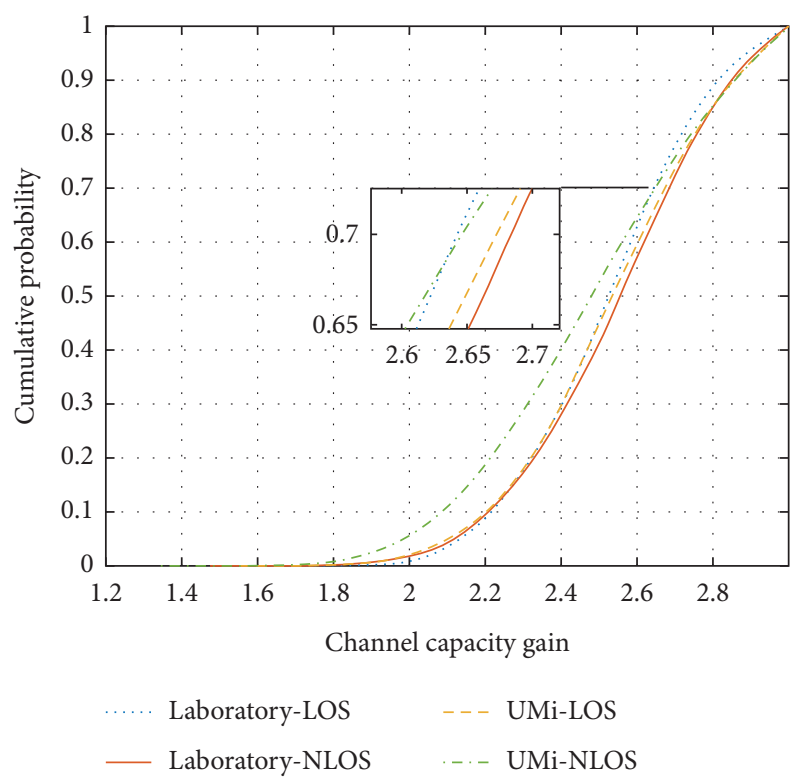

FIgURE 15: The CDFs of $K_{G}$ in the laboratory and the UMi scenario.

TABLE 5: The CDFs of $K_{G}$ in the laboratory and the UMi scenario.

\begin{tabular}{lcccc}
\hline CDF (\%) & Laboratory & \multicolumn{2}{c}{ UMi } & LO \\
\hline 10 & LOS & NLOS & 2.0 & 2.08 \\
50 & 2.22 & 2.21 & 2.4 & 2.48 \\
90 & 2.53 & 2.56 & 2.85 & 2.86 \\
\hline
\end{tabular}

\section{Conclusion}

Tripolarized MIMO antenna is a promising technology to improve the spectrum efficiency for wireless mobile communications, which can provide more degrees of freedom in the propagation. In this paper, we investigate the channel characteristics and evaluate the performance of tripolarized MIMO systems based on channel measurements in realistic environments, i.e., the laboratory and the UMi scenario. The path loss, DS, and XPD for 9 polarization combinations are analyzed and modeled. And, the fitting parameters of statistical results are given. We find that there is a dependency of these large-scale and small-scale parameters on the polarization direction. For example, the path loss discrepancy for different polarization combinations can be over $10 \mathrm{~dB}$ in the $\mathrm{UMi}$ scenario; the mean XPD may be negative between the copolarization channel and the cross-polarization channel. However, the polarization dependency of channel characteristics is not modeled in current standardized channel models. Furthermore, analysis results indicate that 3 independent subchannels can be supported by tripolarized MIMO systems in both the laboratory and the UMi scenario. These results are helpful to model tripolarized MIMO channels and use the tripolarized MIMO antenna.

\section{Data Availability}

The measured data used to support the findings of this study are available partially from the corresponding author upon request.

\section{Conflicts of Interest}

The authors declare that they have no conflicts of interest.

\section{Acknowledgments}

This work was supported by the National Science Fund for Distinguished Young Scholars (no. 61925102), the National Key R\&D Program of China (no. 2020YFB1805002), the National Natural Science Foundation of China (no. 62031019), and the BUPT-CMCC Joint Innovation Center.

\section{References}

[1] K. David and H. Berndt, " $6 \mathrm{G}$ vision and requirements: is there any need for beyond 5G?" IEEE Vehicular Technology Magazine, vol. 13, no. 3, pp. 72-80, 2018.

[2] W. Saad, M. Bennis, and M. Chen, "A vision of $6 \mathrm{G}$ wireless systems: applications, trends, technologies, and open research problems," IEEE network, vol. 34, no. 3, pp. 134-142, 2020. 
[3] Z. Zhang, Y. Xiao, Z. Ma et al., "6G wireless networks: vision, requirements, architecture, and key technologies," IEEE Vehicular Technology Magazine, vol. 14, no. 3, pp. 28-41, 2019.

[4] ITU, "Representative use cases and key network requirements for network 2030," Technical Report, Telecommunication Standardization Sector of ITU, Geneva, Switzerland, 2020.

[5] E. C. Strinati, S. Barbarossa, J. L. Gonzalez-Jimenez, D. Kténas, N. Cassiau, and C. Dehos, "6G: the next frontier," 2019, https://arxiv.org/abs/1901.03239.

[6] P. Zhang, J. Zhang, Q. Qi et al., "Ubiquitous-X: constructing the future 6G networks," SCIENTIA SINICA Informationis, vol. 50, no. 6, pp. 913-930, 2020.

[7] J.-h. Zhang, P. Tang, L. Yu, T. Jiang, and L. Tian, “Channel measurements and models for 6G: current status and future outlook," Frontiers of Information Technology \& Electronic Engineering, vol. 21, no. 1, pp. 39-61, 2020.

[8] A. Goldsmith, S. A. Jafar, N. Jindal, and S. Vishwanath, "Capacity limits of MIMO channels," IEEE Journal on Selected Areas in Communications, vol. 21, no. 5, pp. 684-702, 2003.

[9] J. Zhang, Y. Zhang, Y. Yu, R. Xu, Q. Zheng, and P. Zhang, “3D MIMO: how much does it meet our expectations observed from channel measurements?" IEEE Journal on Selected Areas in Communications, vol. 35, no. 8, pp. 1887-1903, 2017.

[10] M. Shafi, M. Min Zhang, A. L. Moustakas et al., "Polarized MIMO channels in 3-D: models, measurements and mutual information," IEEE Journal on Selected Areas in Communications, vol. 24, no. 3, pp. 514-527, 2006.

[11] Z. Bao, Z. Nie, and X. Zong, "A novel broadband dual-polarization antenna utilizing strong mutual coupling," IEEE Transactions on Antennas and Propagation, vol. 62, no. 1, pp. 450-454, 2013.

[12] W. L. Stutzman and G. A. Thiele, Antenna Theory and Design, John Wiley \& Sons, Hoboken, NJ, USA, 2012.

[13] M. R. Andrews, P. P. Mitra, and R. DeCarvalho, "Tripling the capacity of wireless communications using electromagnetic polarization," Nature, vol. 409, no. 6818, pp. 316-318, 2001.

[14] G. Gupta, B. Hughes, and G. Lazzi, "On the degrees of freedom in linear array systems with tri-polarized antennas," IEEE Transactions on Wireless Communications, vol. 7, no. 7, pp. 2458-2462, 2008.

[15] D. Piao and Y. Wang, "Experimental evaluation of the tri-polarized MIMO channel properties based on a compact multimode antenna," IEEE Access, vol. 7, pp. 67807-67817, 2019.

[16] A. F. Molisch, Wireless Communications, Vol. 34, John Wiley \& Sons, Hoboken, NJ, USA, 2012.

[17] L. Jiang, L. Thiele, and V. Jungnickel, "Polarization rotation evaluation for macrocell MIMO channel," in Proceeding of the 2009 6th International Symposium on Wireless Communication Systems, pp. 21-25, IEEE, Siena, Italy, October 2009.

[18] N. Das, T. Inoue, T. Taniguchi, and Y. Karasawa, "An experiment on MIMO system having three orthogonal polarization diversity branches in multipath-rich environment," in Proceedings of the IEEE 60th Vehicular Technology Conference, VTC2004-Fall, vol. 2, pp. 1528-1532, Los Angeles, CA, USA, September 2004.

[19] N. Prayongpun and K. Raoof, "Correlation effects and channel capacities for MIMO Polarization Diversity," in Proceedings of the 2006 International Conference on Wireless Communications, Networking and Mobile Computing, pp. 1-4, Wuhan, China, September 2006.

[20] A. Habib, "Multiple polarized MIMO with antenna selection," in Proceedings of the 2011 18th IEEE Symposium on Communications and Vehicular Technology in the Benelux (SCVT), pp. 1-8, Ghent, Belgium, November 2011.
[21] F. Quitin, F. Bellens, A. Panahandeh et al., "A time-variant statistical channel model for tri-polarized antenna systems," in Proceedings of the 21st Annual IEEE International Symposium on Personal, Indoor and Mobile Radio Communications, pp. 64-69, Istanbul, Turkey, September 2010.

[22] F. Quitin, C. Oestges, A. Panahandeh, F. Horlin, and P. De Doncker, "Tri-polarized MIMO systems in real-world channels: channel investigation and performance analysis," Physical Communication, vol. 5, no. 4, pp. 308-316, 2012.

[23] D. Piao, L. Yang, Q. Guo, Y. Mao, and Z. Li, "Measurementbased performance comparison of colocated tripolarized loop and dipole antennas," IEEE Transactions on Antennas and Propagation, vol. 63, no. 8, pp. 3371-3379, 2015.

[24] A. Panahandeh, C. Oestges, J.-M. Dricot, F. Horlin, and P. De Doncker, "Tri-polarized spectrum sensing based on an experimental outdoor-to-indoor cognitive-radio scenario," Physical Communication, vol. 5, no. 4, pp. 317-327, 2012.

[25] Z. Ying, Y. Zhang, P. Tang et al., "Experimental performance of the tri-polarized MIMO channel in urban microcell at 4.9 $\mathrm{GHz}$," in Proceedings of the 2020 IEEE/CIC International Conference on Communications in China (ICCC), pp. 675680, Chongqing, China, August 2020.

[26] J. Zhang, P. Tang, L. Tian, Z. Hu, T. Wang, and H. Wang, "6-100 GHz research progress and challenges from a channel perspective for fifth generation (5G) and future wireless communication," Science China Information Sciences, vol. 60, no. 8, Article ID 080301, 2017.

[27] A. Yang, Z. He, C. Xing, Z. Fei, and J. Kuang, "The role of large-scale fading in uplink massive MIMO systems," IEEE Transactions on Vehicular Technology, vol. 65, no. 1, pp. 477-483, 2015.

[28] X. Yu, J. Zhang, M. Haenggi, and K. B. Letaief, "Coverage analysis for millimeter wave networks: the impact of directional antenna arrays," IEEE Journal on Selected Areas in Communications, vol. 35, no. 7, pp. 1498-1512, 2017.

[29] A. Ashikhmin, L. Li, and T. L. Marzetta, "Interference reduction in multi-cell massive MIMO systems with large-scale fading precoding," IEEE Transactions on Information Theory, vol. 64, no. 9, pp. 6340-6361, 2018.

[30] H. L. Bertoni, Radio Propagation for Modern Wireless Systems, Pearson Education, London, UK, 1999.

[31] S. Sun, T. S. Rappaport, M. Shafi, P. Tang, J. Zhang, and P. J. Smith, "Propagation models and performance evaluation for 5G millimeter-wave bands," IEEE Transactions on Vehicular Technology, vol. 67, no. 9, pp. 8422-8439, 2018.

[32] G. R. Maccartney, T. S. Rappaport, S. Sun, and S. Deng, "Indoor office wideband millimeter-wave propagation measurements and channel models at 28 and $73 \mathrm{GHz}$ for ultra-dense 5G wireless networks," IEEE Access, vol. 3, pp. 2388-2424, 2015.

[33] International Telecommunication Union, Guidelines for Evaluation of Radio Interface Technologies for IMT-2020, International Telecommunication Union, Geneva, Switzerland, 2017.

[34] 3GPP, "Study on channel model for frequencies from 0.5 to 100 GHz (Release 15),” 3GPP Technical Report, 3GPP, Sophia Antipolis, France, 2018.

[35] R. Miao, L. Tian, Y. Zheng, P. Tang, F. Huang, and J. Zhang, "Indoor office channel measurements and analysis of propagation characteristics at $14 \mathrm{Ghz}$," in Proceedings of the 2015 IEEE 26th Annual International Symposium on Personal, Indoor, and Mobile Radio Communications (PIMRC), pp. 2199-2203, Hong Kong, China, August 2015.

[36] P. Tang, J. Zhang, H. Tian et al., "Channel measurement and path loss modeling from $220 \mathrm{GHz}$ to $330 \mathrm{GHz}$ for $6 \mathrm{~g}$ wireless 
communications," China Communications, vol. 18, no. 5, pp. 19-32, 2021.

[37] P. Tang, J. Zhang, M. Shafi, P. A. Dmochowski, and P. J. Smith, "Millimeter wave channel measurements and modelling in an indoor hotspot scenario at $28 \mathrm{GHz}$," in Proceedings of the 2018 IEEE 88th Vehicular Technology Conference (VTC-Fall), pp. 1-5, IEEE, Chicago, IL, USA, 2018.

[38] Y. Azar, G. N. Wong, K. Wang et al., "28 Ghz propagation measurements for outdoor cellular communications using steerable beam antennas in New York City," in Proceedings of the 2013 IEEE International Conference on Communications (ICC), pp. 5143-5147, IEEE, Budapest, Hungary, June 2013.

[39] X. Chen and P. S. Kildal, "Theoretical derivation and measurements of the relationship between coherence bandwidth and RMS delay spread in reverberation chamber," in Proceedings of the 2009 3rd European Conference on Antennas and Propagation, pp. 2687-2690, IEEE, Berlin, Germany, March 2009.

[40] L. Rubio, J. Reig, H. Fernández, and V. M. Rodrigo Peñarrocha, "Experimental UWB propagation channel path loss and time-dispersion characterization in a laboratory environment," International Journal of Antennas and Propagation, vol. 2013, Article ID 350167, 7 pages, 2013.

[41] Z. Ying, T. Jiang, P. Tang, J. Zhang, and L. Tian, "analysis of delay characteristics at $4.9 \mathrm{GHz}$ and $28 \mathrm{GHz}$ in an indoor industrial scenario," in Proceedings of the 2020 14th European Conference on Antennas and Propagation (EuCAP), pp. 1-5, IEEE, Copenhagen, Denmark, March 2020.

[42] T. Jiang, J. Zhang, M. Shafi, L. Tian, and P. Tang, "The comparative study of SV model between 3.5 and $28 \mathrm{GHz}$ in indoor and outdoor scenarios," IEEE Transactions on Vehicular Technology, vol. 69, no. 3, pp. 2351-2364, 2019.

[43] X. Zhang, G. Qiu, J. Zhang, L. Tian, P. Tang, and T. Jiang, "Analysis of millimeter-wave channel characteristics based on channel measurements in indoor environments at $39 \mathrm{ghz}$," in Proceedings of the 2019 11th International Conference on Wireless Communications and Signal Processing (WCSP), pp. 1-6, IEEE, Xi'an, China, October 2019.

[44] A. V. Alejos, M. Garcia Sanchez, and I. Cuinas, "Performance analysis of polarization diversity for indoor scenarios at 41.4 $\mathrm{GHz}$ and $61.5 \mathrm{GHz}$," International Journal of Antennas and Propagation, vol. 2012, Article ID 681820, 8 pages, 2012.

[45] P. Kyösti, D. Laselva, L. Hentilä, and T. Jämsä, "Validating IST-WINNER Indoor MIMO radio channel model," in Proceedings of the IST Mobile and Wireless Summit, Mykonos, Greece, June 2006.

[46] R. W. Heath and A. J. Paulraj, "Switching between diversity and multiplexing in MIMO systems," IEEE Transactions on Communications, vol. 53, no. 6, pp. 962-968, 2005.

[47] J. W. Demmel, "The probability that a numerical analysis problem is difficult," Mathematics of Computation, vol. 50, no. 182, p. 449, 1988.

[48] Y. Yu, J. Zhang, P. J. Smith, and P. A. Dmochowski, "Theoretical analysis of 3-D channel spatial correlation and capacity," IEEE Communications Letters, vol. 22, no. 2, pp. 420-423, 2017.

[49] H. C. Lo, D. B. Lin, T. C. Yang, and H. J. Li, "Effect of polarization on the correlation and capacity of indoor MIMO channels," International Journal of Antennas and Propagation, vol. 2012, Article ID 246348, 11 pages, 2012.

[50] J. Zhang, Z. Zheng, Y. Zhang, J. Xi, X. Zhao, and G. Gui, “3d mimo for $5 \mathrm{~g}$ nr: several observations from 32 to massive 256 antennas based on channel measurement," IEEE Communications Magazine, vol. 56, no. 3, pp. 62-70, 2018. 